\title{
A NOTE ON A TRANSPLANTATION THEOREM OF KANJIN AND MULTIPLE LAGUERRE EXPANSIONS
}

\author{
S. THANGAVELU
}

(Communicated by J. Marshall Ash)

\begin{abstract}
By applying a transplantation theorem of Kanjin, a multiplier theorem and a Cesàro summability result are proved for multiple Laguerre expansions. In the one-dimensional case an improved version of the multiplier theorem is obtained.
\end{abstract}

Consider the normalised Laguerre functions $\mathscr{L}_{k}^{\alpha}, \alpha>-1$, on $\mathbb{R}_{+}=(0, \infty)$ defined by

$$
\mathscr{L}_{k}^{\alpha}(t)=\left(\frac{\Gamma(k+1)}{\Gamma(k+\alpha+1)}\right)^{1 / 2} L_{k}^{\alpha}(t) e^{-t / 2} t^{\alpha / 2}
$$

where $L_{k}^{\alpha}(t)$ are the Laguerre polynomials of type $\alpha$. The functions $\left\{\mathscr{L}_{k}^{\alpha}\right\}$ form a complete orthonormal system for $L^{2}\left(\mathbb{R}_{+}\right)$. Recently, in [4] Kanjin studied the mapping properties of the operator $T_{\alpha}^{\beta}$, which is defined as

$$
T_{\alpha}^{\beta} f=\sum_{k=0}^{\infty}\left(f, \mathscr{L}_{k}^{\beta}\right) \mathscr{L}_{k}^{\alpha}
$$

where $(f, g)$ stands for the inner product in $L^{2}\left(\mathbb{R}_{+}\right)$. For the operator $T_{\alpha}^{\beta}$ he has proved the following result.

Theorem 1.1 (Kanjin). Let $\alpha, \beta>-1$ and $\gamma=\min \{\alpha, \beta\}$. If $\gamma \geq 0$ then

$$
\left\|T_{\alpha}^{\beta} f\right\|_{p} \leq C\|f\|_{p} \text { for } 1<p<\infty .
$$

If $-1<\gamma<0$ then (1.3) is valid for $p$ in the interval $(1+\gamma / 2)^{-1}<p<-2 / \gamma$.

The above theorem is called a transplantation theorem for the following reason. Given a bounded sequence $\lambda(k)$ we can define an operator $M_{\lambda}^{\alpha}$ by setting

$$
M_{\lambda}^{\alpha} f=\sum_{k=0}^{\infty} \lambda(k)\left(f, \mathscr{L}_{k}^{\alpha}\right) \mathscr{L}_{k}^{\alpha}
$$

Received by the editors February 25, 1992.

1991 Mathematics Subject Classification. Primary 42C15; Secondary 42C10, 43A55. 
whenever $f$ has the Laguerre expansion

$$
f=\sum_{k=0}^{\infty}\left(f, \mathscr{L}_{k}^{\alpha}\right) \mathscr{L}_{k}^{\alpha}
$$

From the theorem, we can deduce the norm inequality

$$
\left\|M_{\lambda}^{\alpha} f\right\|_{p} \leq C\|f\|_{p}
$$

for any $\alpha$ if we know (1.6) for a particular $\alpha_{0}$. This follows from the identity

$$
T_{\beta}^{\alpha} M_{\lambda}^{\alpha} T_{\alpha}^{\beta} f=M_{\lambda}^{\beta} f .
$$

As an application Kanjin proves the following result concerning $\boldsymbol{M}_{\lambda}^{\alpha}$.

Theorem 1.2 (Kanjin). Let $\lambda(t)$ be a four times differentiable function on $(0, \infty)$ and satisfy

$$
\sup _{t>0}\left|t^{k} \lambda^{(k)}(t)\right| \leq c_{k}
$$

for $k=0,1,2,3,4$. Then (1.6) is true for $1<p<\infty$ if $\alpha \geq 0$ and for $(1+\alpha / 2)^{-1}<p<-2 / \alpha$ if $-1<\alpha<0$.

Theorem 1.2 is deduced by applying the transplantation theorem to the particular case $\alpha=0$, which is proved by Dlugosz in [1]. Now the aim of this note is to prove an improved version of the above multiplier theorem and also to give applications to higher-dimensional Laguerre expansions.

Let $\mathbb{R}_{+}^{n}=\left\{x \in \mathbb{R}^{n}: x_{j} \geq 0\right.$ for all $\left.j\right\}$, and consider for every $\alpha \in \mathbb{R}_{+}^{n}$ and a multi-index $m=\left(m_{1}, m_{2}, \ldots, m_{n}\right)$, the normalised Laguerre functions $\mathscr{L}_{m}^{\alpha}$ on $\mathbb{R}_{+}^{n}$ defined by

$$
\mathscr{L}_{m}^{\alpha}(x)=\prod_{j=1}^{n} \mathscr{L}_{m_{j}}^{\alpha_{j}}\left(x_{j}\right) .
$$

They form a complete orthonormal system for $L^{2}\left(\mathbb{R}_{+}^{n}\right)$, and the Laguerre expansion of a function $f$ in $L^{p}\left(\mathbb{R}_{+}^{n}\right)$ can be written as

$$
f=\sum_{m=0}^{\infty}\left(f, \mathscr{L}_{m}^{\alpha}\right) \mathscr{L}_{m}^{\alpha}
$$

where the sum is extended over all the multi-indices. Expansions of the above type have been studied by Dlugosz [1] when $\alpha$ is a multi-index.

For the above series (2.2) we define the Cesàro means $\sigma_{N}^{\delta}$ of order $\delta$ by the equation

$$
\sigma_{N}^{\delta} f=\frac{1}{A_{N}^{\delta}} \sum_{k=0}^{N} A_{N-k}^{\delta} \sum_{|m|=k}\left(f, \mathscr{L}_{m}^{\alpha}\right) \mathscr{L}_{m}^{\alpha}
$$

where $A_{k}^{\delta}=\Gamma(k+\delta+1) / \Gamma(k+1)$ are the binomial coefficients. Given a function $\lambda$ on $(0, \infty)$ we also define the multiplier operator $M_{\lambda}^{\alpha}$ as

$$
M_{\lambda}^{\alpha} f=\sum_{k=0}^{\infty} \lambda(2 k+n) \sum_{|m|=k}\left(f, \mathscr{L}_{m}^{\alpha}\right) \mathscr{L}_{m}^{\alpha} .
$$

For the operators (2.3) and (2.4) we prove the following two theorems. 
Theorem 2.1. Let $\delta>\frac{1}{2}$. Then the uniform estimates

$$
\left\|\sigma_{N}^{\delta} f\right\|_{p} \leq C\|f\|_{p}
$$

are valid iff $4 n /(2 n+1+2 \delta)<p<4 n /(2 n-1-2 \delta)$.

Theorem 2.2. Assume that the function $\lambda$ satisfies the conditions

$$
\sup _{t>0}\left|t^{k} \lambda^{(k)}(t)\right| \leq c_{k}
$$

for $k=0,1,2, \ldots, \nu$ where $\nu=n+1$ if $n$ is odd and $\nu=n+2$ if $n$ is even. Then for $1<p<\infty$ we have

$$
\left\|M_{\lambda}^{\alpha} f\right\|_{p} \leq C\|f\|_{p} .
$$

In the case $n=1$ we can take $\nu=1$ in the hypothesis and (2.7) is valid for $\frac{4}{3}<p<4$.

A slightly weaker form of Theorem 2.2 is proved in [1] when $\alpha$ is a multiindex. In that version one has $\nu=n+3$ for all $n$. Theorem 2.1 is known when $n=1$ and is due to Gorlich and Markett $[3,5]$.

For the Laguerre series (2.2) we also define the Riesz transforms $R_{j}, j=$ $1,2, \ldots, n$, by the formula

$$
R_{j} f=\sum_{m=0}^{\infty}\left(2 m_{j}+1\right)(2|m|+n)^{-1}\left(f, \mathscr{L}_{m}^{\alpha}\right) \mathscr{L}_{m}^{\alpha}
$$

Riesz transforms for the Hermite and special Hermite expansions have been studied by the author in $[9,12]$. For the above Riesz transforms $(2.8)$ we prove Theorem 2.3. For $1<p<\infty$ all the Riesz transforms $R_{j}$ are bounded on $L^{p}\left(\mathbb{R}_{+}^{n}\right)$.

All three theorems will be proved by appealing to the $n$-dimensional version of Kanjin's transplantation Theorem 1.1. For $\alpha, \beta$ in $\mathbb{R}_{+}^{n}$ we define $T_{\alpha}^{\beta}$ by

$$
T_{\alpha}^{\beta} f=\sum_{m=0}^{\infty}\left(f, \mathscr{L}_{m}^{\beta}\right) \mathscr{L}_{m}^{\alpha} .
$$

Then, for $f$ in $C_{0}^{\infty}\left(\mathbb{R}_{+}^{n}\right)$ and $1<p<\infty$,

$$
\left\|T_{\alpha}^{\beta} f\right\|_{p} \leq C\|f\|_{p} .
$$

This follows from Theorem 1.1 by iteration.

In view of (2.10) Theorems 2.1, 2.2, and 2.3 will follow once we show that they are true in the particular case $\alpha=0$. It will be shown in the next section that the case $\alpha=0$ follows from known results on special Hermite expansions as a special case. The one-dimensional case of Theorem 2.2 when $\alpha=\frac{1}{2}$ will be deduced from the corresponding result on the Hermite expansions. This will be done in the last section.

Consider the functions $\psi_{m}(z)$ on $\mathbb{C}^{n}$ defined by

$$
\psi_{m}(z)=\prod_{j=1}^{n} L_{m_{j}}\left(\frac{1}{2}\left|z_{j}\right|^{2}\right) e^{-\left|z_{j}\right|^{2} / 4}
$$


where $L_{k}(t)$ are the Laguerre polynomials of type 0 . The functions $\psi_{m}(z)$ are called special Hermite functions since they are related to the Hermite function $\Phi_{m}(x)$ on $\mathbb{R}^{n}$. This terminology is due to Strichartz [6]. In fact, one has

$$
\psi_{m}(z)=\int_{\mathbb{R}^{n}} e^{i x \cdot \xi} \Phi_{m}\left(\xi+\frac{y}{2}\right) \Phi_{m}\left(\xi-\frac{y}{2}\right) d \xi
$$

where $z=x+i y, x, y \in \mathbb{R}^{n}$ (see [2]). Given $f$ on $\mathbb{C}^{n}$ we have the special Hermite expansion

$$
f(z)=(2 \pi)^{-n} \sum_{m=0}^{\infty} f \times \psi_{m}(z)
$$

where the twisted convolution $f \times g$ of two functions is defined by

$$
f \times g(z)=\int_{\mathbb{C}^{n}} f(z-w) g(w) e^{(i / 2) \operatorname{Im} z \cdot \bar{w}} d w .
$$

We can also write (3.3) in the form

$$
f(z)=(2 \pi)^{-n} \sum_{k=0}^{\infty} f \times \varphi_{k}^{n-1}(z)
$$

where $\varphi_{k}^{n-1}(z)=L_{k}^{n-1}\left(\frac{1}{2}|z|^{2}\right) e^{-|z|^{2} / 4}$. For all these facts we refer to [11].

For the special Hermite expansion let $C_{N}^{\delta}$ be the Cesàro means defined by

$$
C_{N}^{\delta} f=\frac{1}{A_{N}^{\delta}} \sum_{k=0}^{N} A_{N-k}^{\delta} \sum_{|m|=k}\left(f \times \psi_{m}\right) .
$$

Given a function $\lambda$ on $(0, \infty)$ we also define a multiplier transform $T_{\lambda}$ by

$$
T_{\lambda} f=\sum_{k=0}^{\infty} \lambda(2 k+n) \sum_{|m|=k}\left(f \times \psi_{m}\right) .
$$

In [11] we proved

Theorem 3.1. Let $\delta>\frac{1}{2}$. Then for $f$ in $L^{p}\left(\mathbb{C}^{n}\right)$

$$
\left\|C_{N}^{\delta} f\right\|_{p} \leq C\|f\|_{p}
$$

holds if and only if $4 n /(2 n+1+2 \delta)<p<4 n /(2 n-1-2 \delta)$.

Regarding $T_{\lambda}$ we have proved the following multiplier theorem in [10].

Theorem 3.2. Let $\lambda$ satisfy the hypothesis of Theorem 2.2. Then for $1<p<\infty$ one has $\left\|T_{\lambda} f\right\|_{p} \leq C\|f\|_{p}$.

The case $\alpha=0$ of Theorems 2.1 and 2.2 will be deduced from the above theorems in the following way. When $f$ is a radial function the twisted convolution $f \times \varphi_{k}^{n-1}$ becomes

$$
f \times \varphi_{k}^{n-1}(z)=\frac{k !(n-1) !}{(k+n-1) !}\left(\int_{0}^{\infty} f(r) \varphi_{k}^{n-1}(r) r^{2 n-1} d r\right) \varphi_{k}^{n-1}(z)
$$


where $\varphi_{k}^{n-1}(r)=\varphi_{k}^{n-1}(z)$ with $|z|=r$. If $f$ is a polyradial function, i.e., $f\left(z_{1}, \ldots, z_{n}\right)=f\left(r_{1}, \ldots, r_{n}\right), r_{j}=\left|z_{j}\right|$, then in view of (3.8) and (3.1) one has

$$
f \times \psi_{m}=\left\{\int_{\mathbb{R}_{+}^{n}} f\left(r_{1}, \ldots, r_{n}\right)\left(\prod_{j=1}^{n} \mathscr{L}_{m_{j}}\left(\frac{1}{2} r_{j}^{2}\right)\right) r_{1} \cdots r_{n} d r_{1} \cdots d r_{n}\right\} \psi_{m} .
$$

Therefore, one sees that

$$
f \times \psi_{m}(\sqrt{2} z)=\left(g, \mathscr{L}_{m}\right) \mathscr{L}_{m}(r)
$$

where $g\left(r_{1}, \ldots, r_{n}\right)=f\left(\sqrt{2 r_{1}}, \ldots, \sqrt{2 r_{n}}\right)$. Therefore, $C_{N}^{\delta} f$ becomes $\sigma_{N}^{\delta} g$ and $T_{\lambda} f$ becomes $M_{\lambda}^{0} g$; hence, the case $\alpha=0$ of Theorems 2.1 and 2.2 follow.

The case $\alpha=0$ of Theorem 3.3 follows from the fact (see [12]) that the Riesz transforms

$$
S_{j} f=\sum_{m=0}^{\infty}\left(2 m_{j}+1\right)(2|m|+n)^{-1} f \times \psi_{m}
$$

for the special Hermite expansions are bounded on $L^{p}\left(\mathbb{C}^{n}\right), 1<p<\infty$.

\section{4}

Consider the normalised Hermite functions $h_{k}(x)$ on $\mathbb{R}$. We also consider the Laguerre function $\varphi_{k}^{\alpha}$ of another type defined by, for $\alpha$ real,

$$
\varphi_{k}^{\alpha}(x)=\mathscr{L}_{k}^{\alpha}\left(x^{2}\right)(2 x)^{1 / 2}, \quad x \in \mathbb{R}_{+} .
$$

Then the Hermite functions $h_{k}$ and $\varphi_{k}^{\alpha}$ are related by (see [7])

$$
h_{2 k}(x)=(-1)^{k} \frac{1}{\sqrt{2}} \varphi_{k}^{-1 / 2}(x), \quad h_{2 k+1}(x)=(-1)^{k} \frac{1}{\sqrt{2}} \varphi_{k}^{1 / 2}(x) .
$$

Consider a multiplier transform $M$ for the Hermite series defined by

$$
M f(x)=\sum_{k=0}^{\infty} \lambda(k)\left(f, h_{k}\right) h_{k}(x)
$$

In [8] we proved

Theorem 4.1. Assume that $\lambda$ is bounded and satisfies $\left|t \lambda^{\prime}(t)\right| \leq C$ for all $t>0$. Then $M$ is bounded on $L^{p}(\mathbb{R}), 1<p<\infty$.

Since $h_{2 k}$ is even and $h_{2 k+1}$ is odd, by considering $f$ to be odd we see that

$$
M f(x)=\sum_{k=0}^{\infty} \lambda(2 k+1)\left(f, \varphi_{k}^{1 / 2}\right) \varphi_{k}^{1 / 2}(x),
$$

and this is related to $M_{\lambda}^{1 / 2}$ in the following way. An easy calculation shows that

$$
\left(f, \varphi_{k}^{1 / 2}\right)=\frac{1}{\sqrt{2}}\left(g, \mathscr{L}_{k}^{1 / 2}\right)
$$


where $f(\sqrt{x}) x^{-1 / 4}=g(x)$. Therefore,

$$
M f(\sqrt{x}) x^{-1 / 4}=2 \sum_{k=0}^{\infty} \lambda(2 k+1)\left(g, \mathscr{L}_{k}^{1 / 2}\right) \mathscr{L}_{k}^{1 / 2}(x) .
$$

If we know that for $\frac{4}{3}<p<4$

$$
\int_{0}^{\infty}|M f(x)|^{p} x^{-p / 2+1} d x \leq C \int_{0}^{\infty}|f(x)|^{p} x^{-p / 2+1} d x
$$

then it follows that

$$
\int_{0}^{\infty}\left|M_{\lambda}^{1 / 2} g(x)\right|^{p} d x \leq C \int_{0}^{\infty}|g(x)|^{p} d x
$$

hence, the case $n=1, \alpha=\frac{1}{2}$ of Theorem 2.2 follows. We claim that (4.7) is true.

To prove the claim we recall the proof of Theorem 4.1. Let $T^{t}$ be the semigroup on $L^{p}(\mathbb{R})$ defined by

$$
T^{t} f=\sum_{k=0}^{\infty} e^{-(2 k+1) t}\left(f, h_{k}\right) h_{k}
$$

For this semigroup we defined the $g$ and $g^{*}$ functions in the following way:

$$
(g(f, x))^{2}=\int_{0}^{\infty} t\left|\partial_{t} T^{t} f(x)\right|^{2} d t
$$

$$
\left(g^{*}(f, x)\right)^{2}=\int_{-\infty}^{\infty} \int_{0}^{\infty} t^{1 / 2}\left(1+t^{-1 / 2}|x-y|\right)^{-2}\left|\partial_{t} T^{t} f(y)\right|^{2} d y d t
$$

For the $g$ and $g^{*}$ functions we proved that

$$
\begin{gathered}
C_{1}\|f\|_{p} \leq\|g(f)\|_{p} \leq C_{2}\|f\|_{p}, \quad 1<p<\infty, \\
\left\|g^{*}(f)\right\|_{p} \leq C\|f\|_{p}, \quad p>2 .
\end{gathered}
$$

Under the assumption that $\left|t \lambda^{\prime}(t)\right|$ is bounded we verified that

$$
g(M f, x) \leq C g^{*}(f, x),
$$

and in view of (4.12) and (4.13) this proved Theorem 4.1.

Therefore, in order to prove the weighted version we need to check that

$$
\begin{gathered}
C_{1}\|f\|_{p, w} \leq\|g(f)\|_{p, w} \leq C_{2}\|f\|_{p, w}, \quad \frac{4}{3}<p<4, \\
\left\|g^{*}(f)\right\|_{p, w} \leq C\|f\|_{p, w}, \quad 2<p<4,
\end{gathered}
$$

where $\|f\|_{p, w}$ stands for the norm

$$
\|f\|_{p, w}=\left(\int_{-\infty}^{\infty}|f(x)|^{p}|x|^{-p / 2+1} d x\right)^{1 / p} .
$$

Thus we need weighted norm inequalities for the $g$ and $g^{*}$ functions.

In [8] we proved the $L^{p}$ boundedness of $g$ by applying singular integral theory. We identified $g$ with a singular integral operator whose kernel takes 
values in the Hilbert space $L^{2}\left(\mathbb{R}_{+}, t d t\right)$. When the weight function $w$ is in the Muckenhoupt class $A_{p}$ (see [13]) then we also have

$$
\int_{-\infty}^{\infty}|g(f)|^{p} w(x) d x \leq C \int_{-\infty}^{\infty}|f(x)|^{p} w(x) d x
$$

When $\frac{4}{3}<p<4, w(x)=|x|^{-p / 2+1}$ is in $A_{p}$; hence, the right-hand side inequality of $(4.12)^{\prime}$ is valid. We will now show that the reverse inequality is also valid.

From [8] we recall that we have the partial isometry

$$
\|g(f)\|_{2}=\frac{1}{2}\|f\|_{2}
$$

from this, by polarisation, we obtain

$$
\left|\int_{-\infty}^{\infty} f_{1}(x) \bar{f}_{2}(x) d x\right|=4 \int_{-\infty}^{\infty} \int_{0}^{\infty} t \partial_{t} T^{t} f_{1}(x) \overline{\partial_{t} T^{t} f_{2}(x)} d t d x .
$$

This gives the inequality

$$
\left|\int_{-\infty}^{\infty} f_{1}(x) \bar{f}_{2}(x) d x\right| \leq 4 \int_{-\infty}^{\infty} g\left(f_{1}, x\right) g\left(f_{2}, x\right) d x .
$$

Let us now take $h(x)=f_{2}(x)|x|^{-1 / 2+1 / p}$ so that

$$
\begin{aligned}
& \left.\left|\int_{-\infty}^{\infty} f_{1}(x)\right| x\right|^{-1 / 2+1 / p} \bar{f}_{2}(x) d x \mid \\
& \quad \leq 4 \int_{-\infty}^{\infty} g\left(f_{1}, x\right)|x|^{-1 / 2+1 / p} g(h, x)|x|^{-1 / 2+1 / q} d x
\end{aligned}
$$

where $q$ is the index conjugate to $p$. An application of Holder's inequality gives

$$
\begin{aligned}
& \int_{-\infty}^{\infty} g\left(f_{1}, x\right)|x|^{-1 / 2+1 / p} g(h, x)|x|^{-1 / 2+1 / q} d x \\
& \quad \leq\left(\int_{-\infty}^{\infty}\left|g\left(f_{1}, x\right)\right|^{p}|x|^{-p / 2+1} d x\right)^{1 / p}\left(\int_{-\infty}^{\infty}|g(h, x)|^{q}|x|^{-q / 2+1} d x\right)^{1 / q} .
\end{aligned}
$$

Applying the direct inequality $(4.12)^{\prime}$ to the second factor we get

$$
\begin{aligned}
\int_{-\infty}^{\infty}|g(h, x)|^{q}|x|^{-q / 2+1} d x & \leq C \int_{-\infty}^{\infty}\left|f_{2}(x)\right|^{q}|x|^{-q / 2+q / p+1-q / 2} d x \\
& \leq C \int_{-\infty}^{\infty}\left|f_{2}(x)\right|^{q} d x .
\end{aligned}
$$

In view of (4.20) and (4.21) the inequality (4.19) becomes

$$
\left.\left|\int_{-\infty}^{\infty} f_{1}(x)\right| x\right|^{-1 / 2+1 / p} \bar{f}_{2}(x) d x \mid \leq C\left\|g\left(f_{1}\right)\right\|_{p, w}\left\|f_{2}\right\|_{q} .
$$

Taking the supremum over all $f$ with $\left\|f_{2}\right\|_{q} \leq 1$ we obtain

$$
\int_{-\infty}^{\infty}\left|f_{1}(x)\right|^{p}|x|^{-p / 2+1} d x \leq C\left\|g\left(f_{1}\right)\right\|_{p, w} .
$$

This completes the proof of $(4.12)^{\prime}$. 
To establish the inequality $(4.13)^{\prime}$ we observe that

$$
\int_{-\infty}^{\infty}\left(g^{*}(f, x)\right)^{2} h(x) d x \leq \int_{-\infty}^{\infty}(g(f, x))^{2} \Lambda h(x) d x
$$

for every nonnegative function $h$ where $\Lambda h$ is the Hardy-Littlewood maximal function. If $2<p<4$, let $r=p / 2$ and $s$ be the conjugate index of $r$. Setting $h_{1}(x)=h(x)|x|^{-1+1 / r}$ we have

$$
\begin{aligned}
\int_{-\infty}^{\infty} & \left(g^{*}(f, x)\right)^{2}|x|^{-1+1 / r} h(x) d x \\
& \leq C \int_{\infty}^{\infty}(g(f, x))^{2}|x|^{-1+1 / r}|x|^{1 / s} \Lambda h_{1}(x) d x \\
& \leq C\left(\int_{-\infty}^{\infty}(g(f, x))^{p}|x|^{-p / 2+1} d x\right)^{2 / p}\left(\int_{-\infty}^{\infty}|x|\left(\Lambda h_{1}(x)\right)^{s} d s\right)^{1 / s}
\end{aligned}
$$

by an application of Holder's inequality. Since $s>2,|x| \in A_{s}$; hence,

$$
\begin{aligned}
\int_{-\infty}^{\infty}|x|\left(\Lambda h_{1}(x)\right)^{s} d s & \leq C \int_{-\infty}^{\infty}|h(x)|^{s}|x|^{-s+s / r+1} d x \\
& \leq C \int_{-\infty}^{\infty}|h(x)|^{s} d x .
\end{aligned}
$$

Thus we have the inequality

$$
\begin{aligned}
& \int_{-\infty}^{\infty}\left(g^{*}(f, x)\right)^{2}|x|^{-1+1 / r} h(x) d x \\
& \quad \leq C\left(\int_{-\infty}^{\infty}|f(x)|^{p}|x|^{-p / 2+1} d x\right)^{2 / p}\|h\|_{s} .
\end{aligned}
$$

Taking the supremum over all $h$ with $\|h\|_{s} \leq 1$ we obtain

$$
\int_{-\infty}^{\infty}\left(g^{*}(f, x)\right)^{p}|x|^{-p / 2+1} d x \leq C \int_{-\infty}^{\infty}|f(x)|^{p}|x|^{-p / 2+1} d x .
$$

This proves the inequality $(4.13)^{\prime}$.

Therefore, in view of $(4.12)^{\prime},(4.13)^{\prime}$, and (4.14) we obtain the weighted inequality

$$
\int_{-\infty}^{\infty}|M f(x)|^{p}|x|^{-p / 2+1} d x \leq C \int_{-\infty}^{\infty}|f(x)|^{p}|x|^{-p / 2+1} d x
$$

for $\frac{4}{3}<p<4$, and this proves the multiplier theorem for $\alpha=\frac{1}{2}$. By applying the transplantation theorem we complete the proof of Theorem 2.2 when $n=1$.

\section{REFERENCES}

1. D. Dlugosz, $L^{p}$ multipliers for the Laguerre expansions, Colloq. Math. 54 (1987), 287-293.

2. G. Folland, Harmonic analysis in phase space, Ann. of Math. Stud., vol. 112, Princeton Univ. Press, Princeton, NJ, 1989.

3. E. Gorlich and C. Markett, Mean Cesaro summability and operator norms for Laguerre expansions, Comment. Math. Prace Mat. Tomus Specialus II (1979), 139-148.

4. Y. Kanjin, A transplantation theorem for Laguerre series, preprint, 1990. 
5. C. Markett, Norm estimates for Cesaro means of Laguerre expansions, Approximation and Function Spaces (Proc. Conf. Gdansk, 1979), North-Holland, Amsterdam, 1981, pp. 419435.

6. R. Strichartz, Harmonic analysis as spectral theory of Laplacians, J. Funct. Anal. 87 (1989), 51-148.

7. G. Szego, Orthogonal polynomials, Amer. Math. Soc. Colloq. Publ., vol. 23, Amer. Math. Soc., Providence, RI, 1967.

8. S. Thangavelu, Multipliers for Hermite expansions, Mat. Ibero Americana 3 (1987), 1-24.

9. _ Riesz transforms and the wave equation for the Hermite operator, Comm. Partial Differential Equations 15 (1990), 1199-1215.

10. Littlewood-Paley-Stein theory on $\mathbb{C}^{n}$ and Weyl multipliers, Rev. Mat. Ibero Americana 6 (1990), 75-90.

11. __ Bochner-Riesz means, Weyl multipliers and special Hermite expansions, Ark. Mat. 29 (1991), 307-321.

12. __ Conjugate Poisson integrals and Riesz transforms for Hermite and special Hermite expansions, Colloq. Math. 64 (1993), 103-113.

13. A. Torchinsky, Real variable methods in harmonic analysis, Academic Press, London, 1986.

Tata Institute for Fundamental Research Centre, Post Box No. 1234, Indian InstiTUte of Science Campus, Bangalore, INDia

E-mail address: veluma@tifrbng.ernet.in 